\title{
Combination of the CAGE and serum gamma-glutamyl transferase: An effective screening tool for alcohol use disorder and alcohol \\ dependence
}

Young Min Choe ${ }^{1,2}$, Boung Chul Lee ${ }^{2,5}$, Ihn-Geun Choi',3, Guk-Hee Suh',2, Dong Young Lee, Jee Wook Kim 1,2

'Department of Neuropsychiatry, Hallym University Dongtan Sacred Heart Hospital, Hwaseong, Gyeonggi, Republic of Korea;

3epartment of Neuropsychiatry, Hallym University Kangnam Sacred Heart Hospital, Seoul, Republic of Kor

Department or Neuropsychary, Halym Universty Kangnam Sacred Heart Hospla, Seon, Republic of Korea,

${ }^{4}$ Department of Neuropsychiatry, Seoul National University Hospital, Seoul, Republic of Korea,

sDepartment of Neuropsychiatry, Hallym University Hangang Sacred Heart Hospital, Seoul, Republic of Korea.

\section{Objective}

- The CAGE is a convenient test for alcohol-related disorder due to its brevity, but it is not as effective as the alcohol use disorders identification test (AUDIT).

- Gamma-glutamyl transferase (GGT) is an objective blood biochemical marker of excessive alcohol intake; however, it has low sensitivity. This study tested the performance of the combined use of CAGE and GGT to screen problem drinking (PD), alcohol use disorder (AUD), and alcohol dependence (AD).

\section{Materials \& Methods}

- A total of 394 subjects composed of 91 normal controls (NC) and 303 subjects with PD were enrolled in this study. Of the PD subjects, 147 were diagnosed with AUD (77 alcohol abuse and $70 \mathrm{AD})$.

- All subjects underwent a clinical interview including a detailed history of alcohol drinking and the AUDIT and the CAGE.

- Serum GGT levels were determined using a gamma-GT FS kit (DiaSys Diagnostic Systems, Holzheim, Germany) on the Hitachi 7600 automatic analyzer (Tokyo, Japan) using the Szasz-Persijn method.

- A series of multiple logistic regression models for PD, AUD, and AD discrimination were used to obtain new combined CAGE and GGT scores after adjusting for age and gender (CAGE+GGT)

- A receiver operating characteristic curve analysis was conducted to determine how well the CAGE + GGT score discriminated between individuals with PD, AUD, and AD.

\section{Results}

Table 1 Subject characteristics.

\begin{tabular}{|c|c|c|c|c|c|c|c|}
\hline & $\begin{array}{l}\text { All } \\
(\mathrm{n}=394)\end{array}$ & $\begin{array}{l}\text { Non-PD (NC) } \\
(\mathrm{n}=91)\end{array}$ & $\begin{array}{l}P_{(\mathrm{n}=303)} \\
{ }^{\mathrm{PD}}\end{array}$ & $\begin{array}{l}\text { Non-AUD } \\
(\mathrm{n}=247)\end{array}$ & $\begin{array}{l}\text { AUD } \\
(\mathrm{n}=147)\end{array}$ & $\begin{array}{l}\text { Non-AD } \\
(\mathrm{n}=324)\end{array}$ & $\begin{array}{l}\mathrm{AD} \\
(\mathrm{n}=70)\end{array}$ \\
\hline Age (Years) & $42.72+12.33$ & $48.78+14.07$ & $40.89 \pm 11.16$ & $42.46 \pm 13.55$ & $43.15 \pm 10.00$ & $42.16 \pm 13.05$ & $45.30 \pm 7.84$ \\
\hline Gender (MF) & $309 / 85$ & $55 / 36$ & $254 / 49$ & $177 / 70$ & $132 / 15$ & $241 / 83$ & $68 / 2$ \\
\hline First drinking age & $19.611 \pm 4.96$ & $21.71 \pm 7.40$ & $19.01 \pm 3.81$ & $20.09 \pm 5.48$ & $18.81 \pm 3.85$ & $19.88 \pm 5.03$ & $18.39 \pm 4.47$ \\
\hline $\begin{array}{l}\text { Family history } \\
\text { nafo }\end{array}$ & 50/344 & $4 / 87$ & $46 / 257$ & $18 / 229$ & $32 / 115$ & $28 / 296$ & $22 / 48$ \\
\hline AUDIT & $11.63 \pm 9.62$ & $1.76 \pm 1.82$ & $14.59 \pm 9.01$ & $6.54 \pm 4.73$ & $20.18 \pm 9.69$ & $8.24 \pm 6.08$ & $27.30 \pm 7.14$ \\
\hline \multicolumn{8}{|l|}{ AUDIT sub-category } \\
\hline Alcohol consumption & $6.42+3.56$ & $1.63 \pm 1.58$ & $7.86 \pm 2.60$ & $5.00 \pm 3.23$ & $8.81 \pm 2.73$ & $5.62 \pm 3.34$ & $10.14 \pm 1.76$ \\
\hline Alcohol dependence & $1.93 \pm 2.90$ & $0.08 \pm 0.40$ & $2.49 \pm 3.09$ & $0.52 \pm 0.93$ & $4.31 \pm 3.47$ & $0.89 \pm 1.46$ & $6.77 \pm 2.99$ \\
\hline Alcohol related-harm & $3.27 \pm 4.24$ & $0.05 \pm 0.35$ & $4.24 \pm 4.39$ & $1.02 \pm 1.70$ & $7.07 \pm 4.52$ & $1.74 \pm 2.48$ & $10.39 \pm 3.35$ \\
\hline CAGE & $1.56 \pm 1.40$ & $0.35 \pm 0.66$ & $1.93 \pm 1.36$ & $0.73 \pm 0.82$ & $2.97 \pm 0.98$ & $1.17 \pm 1.16$ & $3.39 \pm 0.82$ \\
\hline GGT(IU/L) & $52.79 \pm 74.15$ & $23.13 \pm 14.89$ & $61.70 \pm 82.12$ & $35.79 \pm 38.77$ & $81.37 \pm 104.67$ & $39.89 \pm 42.68$ & $112.48 \pm 135.62$ \\
\hline
\end{tabular}

Table 2 Multiple logistic regression analyses treating alcohol use status as a dependent variable.

\begin{tabular}{|c|c|c|c|c|c|}
\hline $\begin{array}{c}\text { Dependent } \\
\text { variable }\end{array}$ & Independent variable & B & SE & OR $(95 \% \mathrm{CI})$ & $\mathrm{p}$ \\
\hline \multicolumn{6}{|l|}{ PD } \\
\hline & Age & -0.064 & 0.012 & $0.938(0.917-0.961)$ & $<0.001$ \\
\hline & Gender & 0.229 & 0.362 & $1.257(0.619-2.554)$ & 0.527 \\
\hline & CAGE & 1.311 & 0.204 & $3.709(2.485-5.535)$ & $<0.001$ \\
\hline & GGT & 0.027 & 0.010 & $1.027(1.008-1.048)$ & 0.006 \\
\hline \multicolumn{6}{|c|}{ (2) } \\
\hline & Age & 0.010 & 0.014 & $1.010(0.983-1.038)$ & 0.464 \\
\hline & Gender & 0.076 & 0.507 & $1.079(0.399-2.918)$ & 0.881 \\
\hline & CAGE & 2.066 & 0.208 & $7.895(5.251-11.870)$ & $<0.001$ \\
\hline & GGT & 0.005 & 0.003 & $1.005(0.999-1.012)$ & 0.128 \\
\hline \multicolumn{6}{|c|}{ s. } \\
\hline & Age & 0.044 & 0.019 & $1.045(1.007-1.085)$ & 0.019 \\
\hline & Gender & 2.141 & 1.044 & $8.509(1.100-65.856)$ & 0.040 \\
\hline & CAGE & 1.763 & 0.228 & $5.829(3.731-9.106)$ & $<0.001$ \\
\hline & GGT & 0.008 & 0.003 & $1.008(1.003-1.013)$ & 0.003 \\
\hline
\end{tabular}

- Both the CAGE score and GGT level significantly and independently contributed to differentiate PD from NC after adjusting for age and gender, and also contributed to distinguish $\mathrm{AD}$ from non- $\mathrm{AD}$

- In contrast, subjects with AUD were separated from non-AUD subjects using the CAGE, but not GGT level. We used the results of multiple logistic regression analyses treating PD, $\mathrm{AUD}$, and $\mathrm{AD}$ as dependent variables to calculate new combined CAGE and GGT scores after adjusting for age and gender (CAGE+GGT score).

- The equations for the new scores derived from the multiple logistic regressions
Table 3 AUROC and cutoff scores for the AUDIT, CAGE, GGT and the combination of CAGE and GGT.

\begin{tabular}{|c|c|c|c|}
\hline & NC vs. PD & Non-AUD vs. AUD & Non-AD vs. $A D$ \\
\hline \multicolumn{4}{|l|}{ AUDIT } \\
\hline AUROC & 0.973 & 0.893 & 0.970 \\
\hline $\mathrm{SE}$ & 0.00728 & 0.0190 & 0.0106 \\
\hline $95 \% \mathrm{CI}$ & $0.951-0.986$ & $0.858-0.922$ & $0.948-0.984$ \\
\hline Cut off & $>5$ & $>12$ & $>17$ \\
\hline Sen/Spe & $90.76 / 95.60$ & $76.87 / 89.07$ & $91.43 / 91.98$ \\
\hline \multicolumn{4}{|l|}{ CAGE } \\
\hline AUROC & 0.834 & 0.939 & 0.916 \\
\hline $\mathrm{SE}$ & 0.0214 & 0.0124 & 0.0175 \\
\hline $95 \% \mathrm{CI}$ & $0.794-0.869$ & $0.910-0.960$ & $0.884-0.942$ \\
\hline Cut off & $>0$ & $>2$ & $>2$ \\
\hline Sen/Spe & $82.18 / 74.73$ & 73.47/97.98 & $88.57 / 84.26$ \\
\hline $\mathrm{z}$ score/p value & $6.927 /<0.0001^{*}$ & $2.535 / 0.0112^{\dagger}$ & $3.233 / 0.0012^{*}$ \\
\hline \multicolumn{4}{|l|}{ GGT } \\
\hline AUROC & 0.736 & 0.724 & 0.799 \\
\hline $\mathrm{SE}$ & 0.0263 & 0.0264 & 0.0257 \\
\hline $95 \% \mathrm{CI}$ & $0.690-0.779$ & $0.677-0.767$ & $0.756-0.838$ \\
\hline Cut off & $>28$ & $>28$ & $>36$ \\
\hline Sen/Spe & $61.39 / 82.42$ & $74.83 / 62.75$ & $80.00 / 68.21$ \\
\hline z score/p value & $9.482 /<0.0001^{*}$ & $6.285 /<0.0001^{*}$ & $6.529 /<0.0001^{*}$ \\
\hline \multicolumn{4}{|l|}{$\mathrm{CAGE}+\mathrm{GGT}$} \\
\hline AUROC & 0.894 & 0.944 & 0.947 \\
\hline $\mathrm{SE}$ & 0.0175 & 0.0116 & 0.0127 \\
\hline $95 \% \mathrm{CI}$ & $0.859-0.922$ & $0.917-0.965$ & $0.920-0.967$ \\
\hline Logit (case) & $>1.063$ & $>0.098$ & $>-1.281$ \\
\hline Sen/Spe & $79.54 / 83.52$ & $77.55 / 96.76$ & $90.00 / 87.96$ \\
\hline z score/p value & $5.301 /<0.0001^{*}$ & $2.894 / 0.0038^{\dagger}$ & $1.652 / 0.0985$ \\
\hline
\end{tabular}

Notes: The combined CAGE and GGT scores were calculated by equations derived from multiple logistic regression analyses after adjusting for age and gender. $Z$
corerpp-value resulted from pairwise comparisons of AUROC (AUDIT vs. others). Bonferroni-corrected P $<0.0167$ (0.05/3) was applied for statisitical significance.

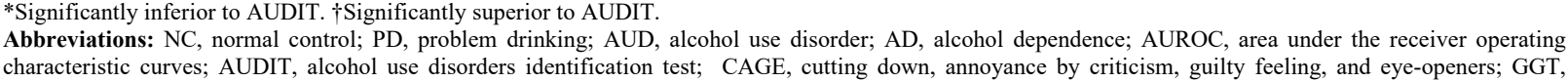
gamma-glutamyl transferase; CAGE+GGT, combination of CAGE and GGT. SE, standard error; CI, Confidence Interval; Sen/Spe, sensitivity/specificity;y Pr (care

Figure 1 ROC curves of AUDIT, CAGE, GGT, and CAGE and GGT combination.

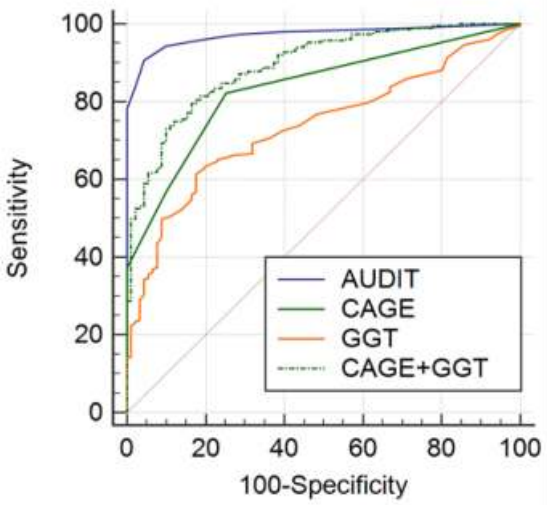

(A) NC vs. PD

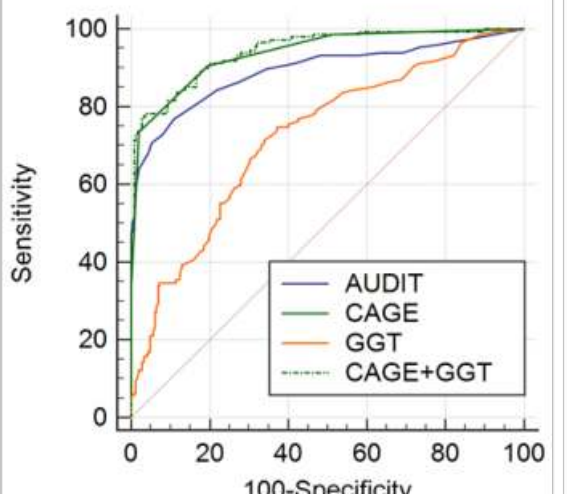

(B) Non-AUD vs. AUD

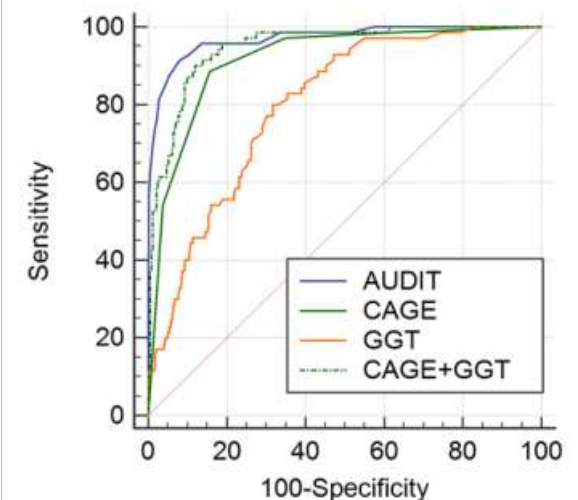

(C) Non-AD vs. AD

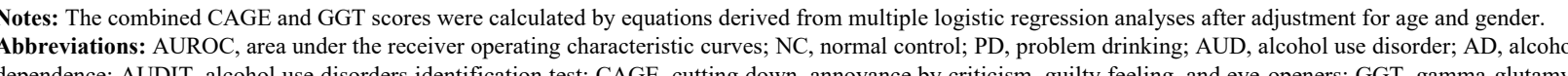
lependence; AUDIT, alcohol use disorders identification

- The ROC curve comparisons for NC vs. PD, non-AUD vs. AUD, and non-AD vs. AD are demonstrated in the following sections. The AUROC of the AUDIT score was 0.973 , indicating good discrimination of PD subjects from $\mathrm{NC}$ individuals.

- The discrimination accuracy of the AUDIT score was significantly better than those of the CAGE score, GGT level, and the CAGE+GGT score. The CAGE score and the CAGE+GGT score were better than the AUDIT score to discriminate AUD.

- No significant difference was found between the CAGE and the CAGE+GGT scores when distinguishing AUD from non-AUD.

- The AUROC of the AUDIT score was 0.970, indicating good discrimination of AD subjects from non-AD individuals.

- The discrimination accuracy of the AUDIT score was significantly better than that of the CAGE score and GGT level, but the CAGE+GGT score was comparable to the AUDIT score for discriminating $\mathrm{AD}$.

- The discrimination accuracy of the AUDIT for PD was significantly better than that of the CAGE or the CAGE+GGT. The CAGE and the CAGE+GGT were better than the AUDIT at discriminating AUD.

- The discrimination accuracy of the AUDIT for AD was significantly better than that of the CAGE and GGT, but the CAGE+GGT was comparable with the AUDIT.

\section{Conclusion}

- Our findings support the combined use of the CAGE questionnaire and serum GGT level as a sensitive and useful tool for AD screening. 